\title{
Reply to comment on: Høi-Hansen T, Pedersen-Bjergaard U, Thorsteinsson B (2005) The Somogyi phenomenon revisited using continuous glucose monitoring in daily life. Diabetologia 48:2437-2438
}

Received: 29 March 2006 / Accepted: 30 March 2006 / Published online: 20 May 2006

(C) Springer-Verlag 2006

To the Editor: Thank you for giving us the opportunity to respond to the letter from Iseda et al. [1]. We certainly acknowledge the extensive scientific contribution made by Dr Somogyi [2]. He concluded, amongst other things, after studying five patients that 'excessive glycosuria (hyperglycemia) is an aftermath of hypoglycemia' [3]. With the years, this phrase has been interpreted as nocturnal hypoglycaemia inducing high morning blood glucose values [4]. In our letter we rejected this clinical statement by showing that if patients have a nocturnal hypoglycaemic episode during the night there was a high probability that blood glucose the following morning was low [5]. This finding suggests that hormonal counter-regulation alone is not capable of inducing hyperglycaemic excursions in type 1 diabetic patients. Iseda et al. now raise the question of whether hyperglycaemia occurs later on in the day after a nocturnal hypoglycaemic episode. In this context it should be noted that the glycaemic response during the day after a night with hypoglycaemia is a complex result of carbohydrate intake (sometimes increased by the hypoglycaemic episode), insulin dosage (possibly reduced if morning glucose is low), prolonged action of counter-regulatory hormones (primarily cortisol and growth hormone), physical activity and other factors. This means that a hyperglycaemic excursion after an episode of nocturnal

\footnotetext{
T. Høi-Hansen $(\bowtie) \cdot$ U. Pedersen-Bjergaard $\cdot$ B. Thorsteinsson Endocrinology Section, Division of Internal Medicine I,

Hillerød Hospital, Helsevej 2,

3400 Hillerød, Denmark

e-mail: thoh@fa.dk

Tel.: +45-4-8294817

Fax: $+45-4-8294783$
}

hypoglycaemia may be caused by reasons other than pure counter-regulatory insulin resistance. Nevertheless, we consider the question to be of clinical importance and have looked further into our data by studying hourly glucose values determined by a continuous glucose monitoring system (CGMS) for $4 \mathrm{~h}$ post-breakfast (Fig. 1) and the maximum value during the day.

In our research letter, we reported a significant difference in mean fasting morning blood glucose between nights without hypoglycaemia and the other two categories of glycaemia [5]. Similarly, we found that $1 \mathrm{~h}$ after measurement of fasting morning blood glucose, the mean glucose value for nights without hypoglycaemia (mean \pm SEM: $11.9 \pm 0.3 \mathrm{mmol} / \mathrm{l}$ ) was significantly different from that for possible hypoglycaemic nights $(9.8 \pm 0.6 \mathrm{mmol} / \mathrm{l}$, $p<0.003$, Bonferroni corrected) and that for hypoglycaemic nights $(10.3 \pm 0.4 \mathrm{mmol} / 1, p<0.006$, Bonferroni corrected). After 2, 3 and $4 \mathrm{~h}$ there were no significant differences between the three categories. Maximum glucose values for days after nights without hypoglycaemia, with possible hypoglycaemia and with hypoglycaemia were $16.1 \pm 0.4$, $14.6 \pm 0.6$ and $16.2 \pm 0.4 \mathrm{mmol} / 1 \quad(p=0.98$ and $p=0.81$ for without hypoglycaemia vs possible hypoglycaemia and with hypoglycaemia, respectively). These results show that the glucose concentration levels out after fasting morning blood glucose. They do not support the proposal that high glucose values during late morning are an indicator of previous hypoglycaemic nights. In conclusion, in type 1 diabetes, nocturnal hypoglycaemia should be suspected in the case of low fasting glucose values in the morning. Hyperglycaemia (fasting or later during the day) should not be regarded as an indicator of previous nocturnal hypoglycaemic episodes. 


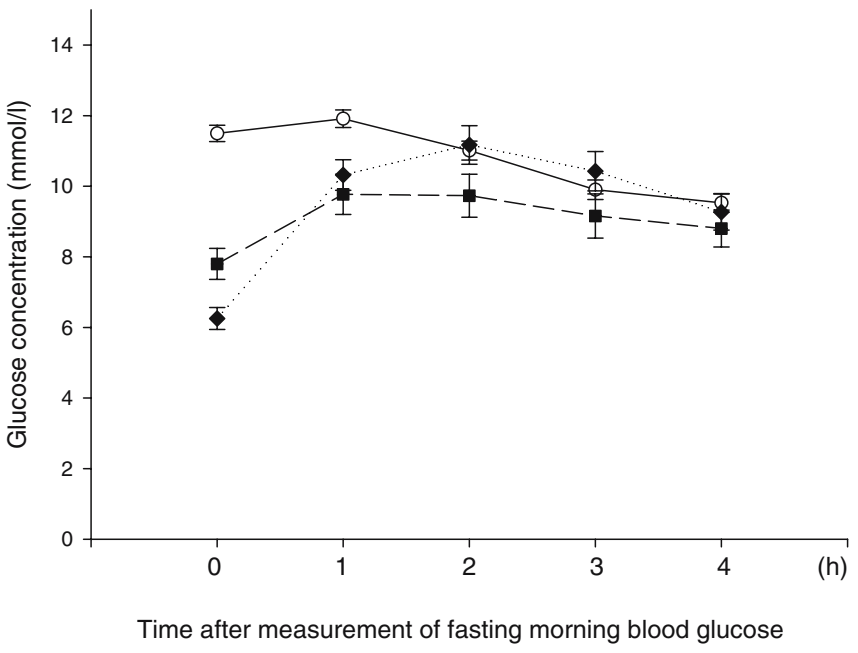

Fig. 1 Fasting morning glucose concentrations measured by selfmonitored blood glucose and CGMS, and hourly post-breakfast glucose concentrations measured by CGMS for $4 \mathrm{~h}$. The data are shown as means \pm SEM. Black diamonds, with nocturnal hypoglycaemia (nadir $\mathrm{CGMS}=2.2 \mathrm{mmol} / \mathrm{l}$ ); black squares, possible nocturnal hypoglycaemia (nadir $\mathrm{CGMS}=2.3-3.5 \mathrm{mmol} / \mathrm{l}$ ); open circles, without nocturnal hypoglycaemia (nadir CGMS $>3.5 \mathrm{mmol} / \mathrm{l}$ )

\section{References}

1. Iseda I, Lins PE, Adamson U, Kollind M (2006) Comment to: Hoi-Hansen T, Pedersen-Bjergaard U, Thorsteinsson B (2005) The Somogyi phenomenon revisited using continuous glucose monitoring in daily life. Diabetologia 48:2437-24-38. Diabetologia. DOI:10.1007/s00125-006-0238-z

2. Walker H Jr (1972) Michael Somogyi, Ph.D. (1883-1971) Metabolism 21:589-590

3. Somogyi M (1959) Exacerbation of diabetes by excess insulin action. Am J Med 26:169-191

4. Raskin P (1984) The Somogyi phenomenon. Sacred cow or bull? Arch Intern Med 144:781-787

5. Høi-Hansen T, Pedersen-Bjergaard U, Thorsteinsson B (2005) The Somogyi phenomenon revisited using continuous glucose monitoring in daily life. Diabetologia 48:2437-2438 